\title{
Distinct Mechanisms of Platelet Aggregation as a Consequence of Different Shearing Flow Conditions
}

\author{
Shinya Goto, Yasuo Ikeda, Enrique Saldívar, and Zaverio M. Ruggeri \\ Roon Research Center for Arteriosclerosis and Thrombosis, Division of Experimental Hemostasis and Thrombosis, Department of \\ Molecular and Experimental Medicine and Department of Vascular Biology, The Scripps Research Institute, La Jolla, California 92037
}

\begin{abstract}
Platelet aggregation contributes to arresting bleeding at wound sites, but may cause occlusion of atherosclerotic vessels, thus curtailing blood flow to vital organs. According to current dogma, the integrin $\alpha_{\mathrm{II}} \beta_{3}$ plays an exclusive role in linking platelets to one another through interactions with fibrinogen or vWf. We demonstrate here that, depending on shearing flow conditions, this process may require vWf binding to glycoprotein Ib $\alpha$, even when $\alpha_{\text {IIb }} \beta_{3}$ is competent to bind adhesive ligands. Platelet activation induced solely by high shear stress is initiated by glycoprotein Ib $\alpha$ interaction with vWf, but results in aggregation only if the latter can bind concurrently to $\alpha_{\text {IIb }} \beta_{3}$. In contrast, platelets exposed to high shear rate after activation by exogenous agonists such as ADP and epinephrine can aggregate when fibrinogen is the $\alpha_{\mathrm{IIb}} \beta_{3}$ adhesive ligand, yet only if $\mathrm{vWf}$ binding to glycoprotein Ib $\alpha$ can also occur. Thus, the latter interaction appears to provide a bond with biomechanical properties necessary to overcome the effects of high shear rate and initiate interplatelet cohesion. These findings highlight the distinct function of two adhesive receptors mediating platelet aggregation under varying fluid dynamic conditions, and modify the current interpretation of a crucial event in hemostasis and thrombosis. (J. Clin. Invest. 1998. 101:479-486.) Key words: hemostasis • thrombosis • von Willebrand factor $\bullet$ fibrinogen $\bullet$ rheology
\end{abstract}

\section{Introduction}

Platelet aggregation is a key mechanism for normal hemostasis limiting blood loss after tissue trauma $(1,2)$, but may lead to arterial occlusion in the setting of atherosclerosis and precipitate diseases such as myocardial infarction $(3,4)$. The first platelet response to vascular injury is adhesion to exposed reactive surfaces, followed (as presently understood) by aggrega-

Address correspondence to Zaverio M. Ruggeri, M.D., The Scripps Research Institute, SBR-8, 10550 N. Torrey Pines Road, La Jolla, CA 92037. Phone: 619-784-8950; FAX: 619-784-2026; E-mail: ruggeri@ scripps.edu

Shinya Goto's present address is First Department of Internal Medicine, Tokai University School of Medicine, Isehara, Japan; Yasuo Ikeda's present address is Department of Internal Medicine, Keio University School of Medicine, Tokyo, Japan.

Received for publication 19 June 1997 and accepted in revised form 14 November 1997.

J. Clin. Invest.

(C) The American Society for Clinical Investigation, Inc. 0021-9738/98/01/0479/08 \$2.00

Volume 101, Number 2, January 1998, 479-486

http://www.jci.org tion of platelets linked to one another by fibrinogen or $\mathrm{vWf}$ bound to the activated GP IIb-IIIa complex (5), now more commonly designated as integrin $\alpha_{\mathrm{II}} \beta_{3}$. In experimental conditions, interplatelet cohesion also occurs when vWf binds to GP Ib $\alpha$ in the presence of the bacterial glycopeptide ristocetin (6) or the snake protein botrocetin (7). This process, however, is of uncertain biological significance because it requires soluble modulators without known counterparts in mammalian organisms, and is more akin to passive agglutination independent of active platelet metabolism than to aggregation. On the other hand, it has been recognized that vWf binding to GP $\mathrm{Ib} \alpha$, whether in the presence $(8)$ or absence $(9,10)$ of exogenous modulators, is coupled to activating signals that may result in the binding of adhesive proteins to $\alpha_{\text {IIb }} \beta_{3}$. Such a mechanism can explain aggregation initiated by vWf under the influence of high shear stress $(11,12)$ as well as by asialo-vWf (13) or mutant type IIB vWf (14) at lower shear stress levels. Thus, the vWf-GP Ib $\alpha$ interaction may induce aggregation, but there is no evidence that it directly mediates platelet-platelet contact in biologically relevant situations. Here we demonstrate a role for $\mathrm{vWf}$ and GP Ib $\alpha$ in interplatelet cohesion, necessary to complement the function of activated $\alpha_{\text {IIb }} \beta_{3}$ under the effect of elevated fluid dynamic forces.

\section{Methods}

Blood collection and platelet preparation. Blood containing as anticoagulant D-phenylalanyl-L-prolyl-L-arginine chloromethyl ketone dihydrochloride (PPACK; Bachem Bioscience Inc., King of Prussia, PA), an inhibitor of $\alpha$-thrombin not affecting divalent cation concentrations (12), was used both in perfusion studies (15) and for obtaining platelet-rich plasma by centrifugation (12). Washed platelets were prepared as described elsewhere (16). All experiments involving human volunteers were performed with approval by the appropriate Institutional Review Committee and in accordance with the Declaration of Helsinki.

Preparation and characterization of purified proteins. Fibrinogen and $v W f$ were purified and characterized as reported previously (17, 18). Asialo-vWf was obtained by treatment with neuraminidase (13). The methods used for the preparation and characterization of fibrinogen and vWf labeled with FITC (Calbiochem-Novabiochem Intl., La Jolla, CA) have been published in detail (16). The monoclonal antibodies used for selective platelet receptor blockade as well as the procedures for IgG purification and preparation of monovalent Fab fragment by papain digestion have been described elsewhere (16). In particular, two anti- $\alpha_{\mathrm{IIb}} \beta_{3}$ antibodies were used: LJ-CP8 and LJ-P5. LJ-CP8 inhibits receptor interaction with both fibrinogen and vWf among other ligands $(18,19)$, while LJ-P5 selectively inhibits vWf binding to $\alpha_{\mathrm{II}} \beta_{3}$ without affecting fibrinogen binding, as documented in previous publications $(16,20,21)$. The anti-vWf antibody NMC-4 (22), directed against the A1 domain of the molecule (23) that contains the GP Ib $\alpha$-binding site $(24,25)$, was a gift of Dr. Akira Yoshioka (Nara Medical College, Nara, Japan).

Shear stress-induced aggregation and ligand binding to platelets. Platelet-rich plasma or washed platelet suspensions were exposed to selected shear rates by varying the speed of the rotating cone in a 
computer-controlled cone-and-plate viscometer at room temperature $\left(22-25^{\circ} \mathrm{C}\right)$ for $6 \mathrm{~min}$. Aggregation was monitored continuously (12). FITC-labeled ligand binding to activated platelets was measured before and after exposure to shear stress using flow cytometric analysis as previously reported in detail for vWf binding (16).

Flow chamber perfusion studies. Platelet thrombus formation onto collagen type I-coated surfaces was evaluated in a modified HeleShaw flow chamber as described $(15,26)$ using an epifluorescence confocal microscope (Axiovert 135M; Carl Zeiss, Inc., Thornwood, $\mathrm{NY}$ ). Platelets were labeled with $10 \mu \mathrm{M}$ mepacrine (quinacrine dihydrochloride; Sigma Chemical Co., St. Louis, MO), a fluorescent dye that accumulates in the dense granules. Leukocytes were labeled concurrently, but did not show any surface interactions under the conditions tested, nor did they interfere with the measurement of platelet thrombi. Moreover, they could easily be identified on the basis of morphologic criteria. Platelet adhesion and thrombus growth were evaluated in real time using a silicon-intensified videocamera (Model C2400; Hamamatsu Photonic Systems Corp., Bridgewater, NJ) and recorded on tape with a videocassette recorder (Magnavox; Philips, Eindhoven, The Netherlands). At selected time points, thrombi were visualized in three dimensions by a series of confocal images representing planes at progressively increasing distance from the collagencoated surface covering the entire height of thrombi. The scanning time to generate each image was $2 \mathrm{~s}$, and the separation interval in the $z$ axis was $5 \mu \mathrm{m}$. Image processing and measurement of surface coverage were performed with the Metamorph software package (Universal Imaging Corp., West Chester, PA).

\section{Results}

Influence of shear rate on the aggregation of nonactivated and activated platelets suspended in plasma. Normal platelets suspended in their native plasma and not interacting with any surface were induced to aggregate in the absence of exogenous agonists when the shear rate exceeded 5,400 $\mathrm{s}^{-1}$ (Fig. 1). In agreement with published findings $(11,12)$, this response was inhibited by blocking GP Ib $\alpha$ as well as $\alpha_{\mathrm{IIb}} \beta_{3}$ (see below). Prior platelet stimulation with the combination of ADP and epinephrine resulted in more rapid onset of aggregation that was similar at all shear rates tested between $1,200 \mathrm{~s}^{-1}$ and $10,800 \mathrm{~s}^{-1}$ (Fig. 1), reflecting agonist-induced $\alpha_{\mathrm{IIb}} \beta_{3}$ activation independent of fluid forces. Blocking $\alpha_{\mathrm{IIb}} \beta_{3}$ on these activated platelets using an antibody that inhibits nonselectively binding of all ligands known to interact with the receptor (antibody LJCP8) impaired aggregation under all shearing flow conditions. In contrast, blocking GP Ib $\alpha$ with an antibody that prevents vWf binding (antibody LJ-Ib1) caused only $2 \%$ inhibition of aggregation at $1,200 \mathrm{~s}^{-1}$, increasing progressively to $10 \%$ inhibition at $2,700 \mathrm{~s}^{-1}, 46 \%$ at $5,400 \mathrm{~s}^{-1}, 48 \%$ at $8,100 \mathrm{~s}^{-1}$, and $77 \%$ at $10,800 \mathrm{~s}^{-1}$ (average of two separate experiments). Concurrent blockade of GP Ib $\alpha$ and $\alpha_{\mathrm{IIb}} \beta_{3}$ showed no synergistic effects at low shear rate, but enhanced considerably the inhibition of aggregation at higher shear rate levels (Fig. 1).

Effect of selective $\alpha_{I I} \beta_{3}$ inhibition on the aggregation of nonstimulated and stimulated platelets at different shear rates. An anti- $\alpha_{\mathrm{IIb}} \beta_{3}$ monoclonal antibody that selectively blocks vWf binding with no effect on fibrinogen binding (antibody LJ-P5) markedly inhibited the aggregation observed when normal platelets suspended in their native plasma in the absence of exogenous agonists were exposed to a shear rate of $10,800 \mathrm{~s}^{-1}$. This antibody was at least as effective as LJ-CP8 in blocking high shear stress-induced aggregation, even though the latter blocks not only vWf but also fibrinogen binding to $\alpha_{\mathrm{IIb}} \beta_{3}$. The inhibition of aggregation caused by LJ-P5 was essentially complete, and was only marginally increased by concurrent blockade of GP Ib $\alpha$ (Fig. 2). Platelets activated with the combination of ADP and epinephrine and exposed to the same shear rate of $10,800 \mathrm{~s}^{-1}$ aggregated more rapidly and to a greater extent than nonactivated platelets. In marked contrast to the results seen with nonactivated platelets, antibody LJ-P5 was much less effective than LJ-CP8 in inhibiting the aggregation of activated platelets at a high shear rate. With either anti$\alpha_{\mathrm{IIb}} \beta_{3}$ antibody, however, concurrent blockade of GP Ib $\alpha$ resulted in nearly complete inhibition of aggregation (Fig. 2). The results seen at the lower shear rate of $1,200 \mathrm{~s}^{-1}$ were remarkably different. Aggregation was negligible in the absence
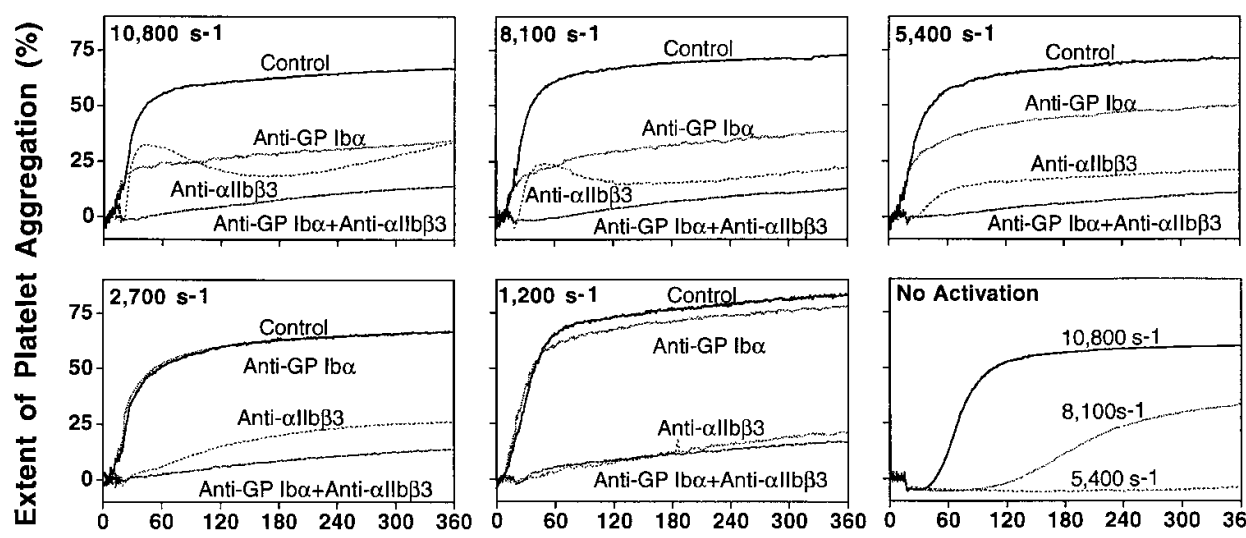

Figure 1. Effect of fluid dynamic forces on platelet aggregation. Platelet-rich plasma was tested without addition of exogenous agonists at three different shear rates (no activation, bottom right) or immediately after activation with ADP and epinephrine, $20 \mu \mathrm{M}$ each, at five dif-
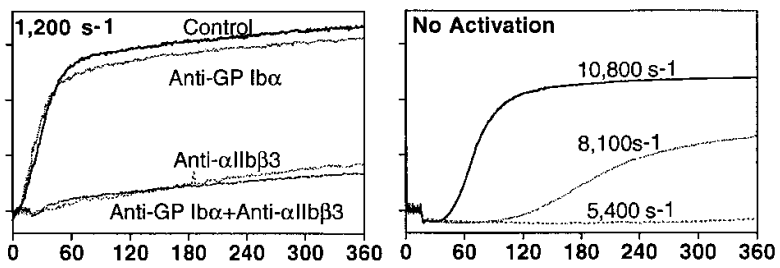
ferent shear rates (remaining five, as indicated). The software controlling the cone-and-plate viscometer always imposed an initial shear rate of $120 \mathrm{~s}^{-1}$ for $15 \mathrm{~s}$, followed by acceleration in $5 \mathrm{~s}$ to the value stated for each experiment. Agonist-stimulated platelets were tested with or without inhibition of GP Ib $\alpha$ and $\alpha_{\text {IIt }} \beta_{3}$ by specific monoclonal antiTime (seconds) bodies (see below). Note that activated platelets exhibited similar extent of aggregation regardless of shear rate and shorter lag phase preceding
aggregation as compared with nonactivated platelets under corresponding conditions. All experimental mixtures contained $400 \mu l$ of plateletrich plasma with $40 \mu \mathrm{l}$ of Hepes-Tyrode buffer, $\mathrm{pH} 7.4$ (16), in the control sample; or $40 \mu \mathrm{l}$ of the anti- $\alpha_{\mathrm{II}} \beta_{3}$ antibody LJ-CP8 (50 $\left.\mu \mathrm{g} / \mathrm{ml}\right) ;$ or $40 \mu \mathrm{l}$ of the anti-GP Ib $\alpha$ antibody LJ-Ib1 (144 $\mu \mathrm{g} / \mathrm{ml})$; or $20 \mu \mathrm{l}$ each of the anti-GP Ib $\alpha$ and anti- $\alpha_{\mathrm{II}} \beta_{3}$ antibodies (144 and $50 \mu \mathrm{g} / \mathrm{ml}$, respectively), as indicated. When needed, agonists were added in a volume of $10 \mu \mathrm{l}$. All reagents were dissolved either in $20 \mathrm{mM} \mathrm{Hepes} \mathrm{with} 150 \mathrm{mM} \mathrm{NaCl}$ or in modified Hepes-Tyrode buffer, $\mathrm{pH}$ 7.4, and the indicated concentrations were those in the final mixtures. The results shown here are representative of two separate experiments that gave comparable results. 
$10,800 \mathrm{~s}^{-1}$

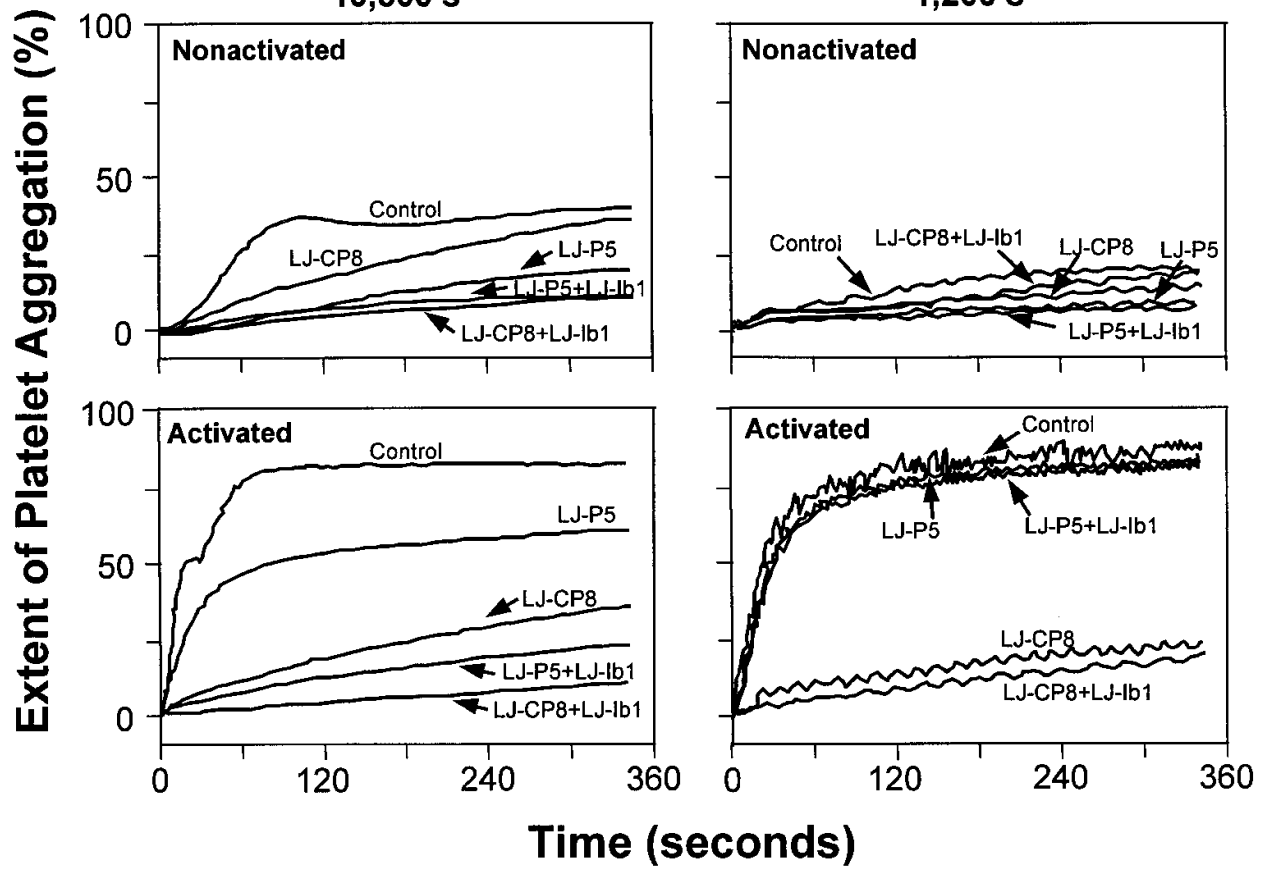

Figure 2. Effect of anti- $\alpha_{\text {II }} \beta_{3}$ antibodies with distinct ligand-blocking selectivity on the aggregation of platelets exposed to different shear rates. These experiments were performed with nonactivated (top) or activated (bottom) platelets suspended in their native plasma as described in the legend to Fig. 1, but a second anti- $\alpha_{\mathrm{II}} \beta_{3}$ antibody, LJ-P5 (final concentration $50 \mu \mathrm{g} / \mathrm{ml}$ ), was used in addition to LJ-CP8. The two antibodies differ for their ability to inhibit soluble ligand binding to $\alpha_{\text {IIb }} \beta_{3}$ since LJ-CP8 blocks receptor interaction with both fibrinogen and vWf, whereas LJ-P5 selectively blocks the interaction with vWf without affecting that with fibrinogen (see Methods). The combined inhibitory effect of anti- $\alpha_{\mathrm{II}} \beta_{3}$ and anti-GP Ib $\alpha$ antibodies was also tested, as indicated (see legend to Fig. 1 for experimental details). Aggregation was evaluated at the two shear rates of 1,200 and $10,800 \mathrm{~s}^{-1}$. The results shown are representative of two separate experiments performed with blood from different donors that gave comparable results. of exogenous agonists, but developed rapidly and was extensive after stimulation with the combination of ADP and epinephrine. This response was obliterated by the anti- $\alpha_{\mathrm{IIb}} \beta_{3}$ antibody blocking vWf and fibrinogen binding (LJ-CP8), whereas the antibody blocking vWf, and not fibrinogen binding (LJP5), allowed aggregation to occur as in the control sample. Addition of the antibody inhibiting GP Ib $\alpha$ function had no influence on the effect, of lack thereof, of the anti- $\alpha_{\mathrm{II}} \beta_{3}$ antibodies (Fig. 2).

Role of fibrinogen and $v W f$ in platelet aggregation at different shear rates. Addition of either purified fibrinogen or $\mathrm{vWf}$ to washed platelets was sufficient to support agonist-induced aggregation at the shear rate of $1,200 \mathrm{~s}^{-1}$. This effect was inhibited by blocking $\alpha_{\text {IIb }} \beta_{3}$, but not GP Ib $\alpha$. At $10,800 \mathrm{~s}^{-1}$, in contrast, only $\mathrm{vWf}$ could mediate maximum aggregation that was inhibited by blocking either $\alpha_{\mathrm{IIb}} \beta_{3}$ or GP Ib $\alpha$, but more effectively by concurrent blockade of both receptors (Fig. 3). The limited aggregation with slow initial onset seen at a high shear rate in the presence of fibrinogen was also inhibited by the anti-GP Ib $\alpha$ antibody (Fig. 3), an indication that it was possibly mediated by released platelet vWf (27). Activated platelets that failed to aggregate significantly at $10,800 \mathrm{~s}^{-1}$ in the presence of fibrinogen, or even if the presence of vWf if GP Ib $\alpha$ was blocked, promptly formed aggregates when the shear rate was decreased to $1,200 \mathrm{~s}^{-1}$. Such a response was inhibited by blocking $\alpha_{\mathrm{IIb}} \beta_{3}$ (Fig. 3), a demonstration that the functional integrity of platelets was not affected by shearing flow.

Washed platelets suspended in buffer exhibited minimal aggregation at a high shear rate in the absence of added soluble vWf, but aggregated promptly when the latter was present. Even prior activation with the combination of ADP and epi- nephrine resulted in just a modest increase in aggregation at a high shear rate unless soluble vWf was present in the suspension. Moreover, the anti-A1 domain antibody NMC-4 markedly inhibited this effect of added vWf, confirming that interaction of the latter with GP Ib $\alpha$ was essential to support the aggregation of washed platelets exposed to a high shear rate, whether or not platelets had been activated by exogenous agonists (Fig. 4). Washed platelets exposed to the higher shear rate of $10,800 \mathrm{~s}^{-1}$ in the absence of added soluble vWf showed increasing aggregation when the shear rate was decreased to $1,200 \mathrm{~s}^{-1}$. The final extent of this response was similar whether activation with exogenous ADP and epinephrine had taken place or not (Fig. 4). These results indicate that ligands released from platelets as a consequence of activation and/or associated with their membrane can support some degree of aggregation, particularly at lower shear rate levels. Nevertheless, additional soluble vWf appears to be essential for significant aggregation to occur at higher shear rates (Fig. 4), presumably reflecting the role of plasma as opposed to platelet $\mathrm{vWf}$ in processes that occur in blood.

Effect of shear rate on platelet aggregation induced by asialo $v W f$. The concept that shear stress-dependent vWf binding to GP Ib $\alpha$ serves essentially to promote activation in the absence of other stimuli $(8,28)$ cannot explain the present findings indicating the inability of soluble agonists to induce aggregation at a high shear rate unless vWf and GP Ib $\alpha$ are also present (Figs. 1-4). Nevertheless, we considered the possibility that unique signaling properties (28) were responsible for the role of the vWf-GP Ib $\alpha$ interaction in aggregation at high shear rates. To test this hypothesis we used asialo-vWf because it activates $\alpha_{\mathrm{IIb}} \beta_{3}$ by binding to GP Ib $\alpha$ without requiring chemical 

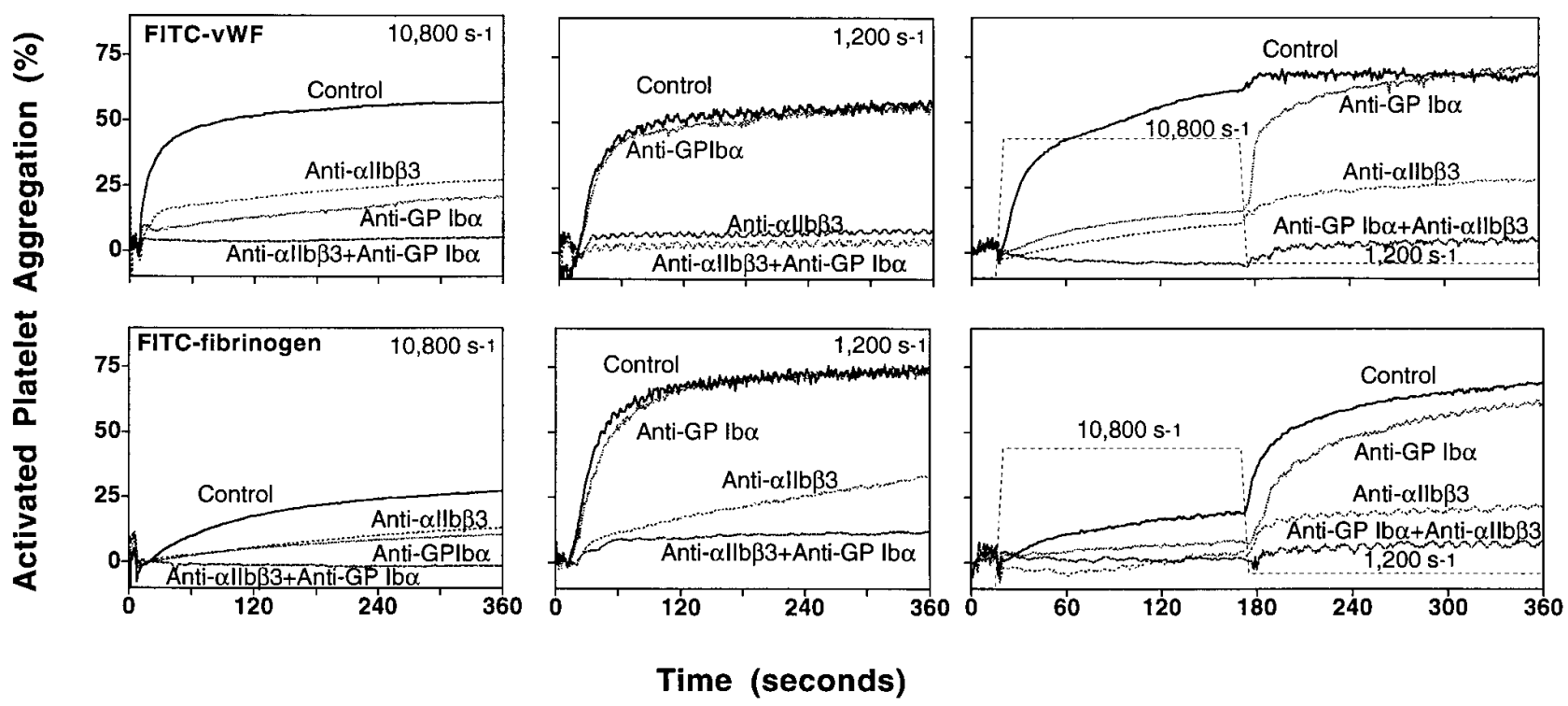

Figure 3. Aggregation of activated washed platelets as a function of shear rate in the presence of vWf or fibrinogen. Washed platelet suspensions were prepared at a count of $2.4 \times 10^{5} / \mu \mathrm{l}$ in modified Hepes-Tyrode buffer, $\mathrm{pH} 7.4$, with the addition of $6.5 \mathrm{mg} / \mathrm{ml} \mathrm{BSA} \mathrm{and} 1.25 \mathrm{mM} \mathrm{CaCl}$. 400 $\mu l$ of the suspension was mixed with $40 \mu l$ of the same buffer in the control sample, or with specific monoclonal antibodies as described in the legend to Fig. 1. After adding $50 \mu \mathrm{l}$ of either FITC-vWf $(15 \mu \mathrm{g} / \mathrm{ml}$; top $)$ or FITC-fibrinogen $(20 \mu \mathrm{g} / \mathrm{ml}$; bottom $)$ and $10 \mu l$ of a solution of ADP and epinephrine $(20 \mu \mathrm{M}$ each), the mixtures were incubated at room temperature for $10 \mathrm{~min}$ in order to reproduce the conditions used to measure ligand binding to platelets not exposed to shear forces (see Fig. 6). All reagents were either in $20 \mathrm{mM}$ Hepes with $150 \mathrm{mM} \mathrm{NaCl}$, or in modified Hepes-Tyrode buffer, $\mathrm{pH}$ 7.4. Aggregation was measured either at constant shear rate $\left(1,200 \mathrm{~s}^{-1}\right.$ or $10,800 \mathrm{~s}^{-1}$ for $\left.6 \mathrm{~min}\right)$ or at $10,800 \mathrm{~s}^{-1}$ for $3 \mathrm{~min}$ followed by $1,200 \mathrm{~s}^{-1}$ for $3 \mathrm{~min}$. The results shown are representative of two separate experiments performed with blood from different donors that gave comparable results. At the higher shear rate, the maximal extent of aggregation (average of the two experiments) was $67 \%$ in the presence of vWf and $32 \%$ in the presence of fibrinogen. The corresponding initial rates of aggregation were 3.26 and $1.12 \%$ per second, respectively. The inhibition of aggregation was between 70 and $80 \%$ when GP Ib $\alpha$ or $\alpha_{\text {IIb }} \beta_{3}$ were blocked individually, but it was $>99 \%$ when the two receptors were blocked concurrently. The concentrations of fibrinogen and vWf used in these experiments were known to be saturating with respect to binding to $\alpha_{\mathrm{IIb}} \beta_{3}(16)$.

or biomechanical modulation (13), and should therefore support aggregation more efficiently than the native counterpart regardless of fluid forces. Addition of asialo-vWf to plateletrich plasma resulted in extensive aggregation at a shear rate of $1,200 \mathrm{~s}^{-1}$, albeit with typical slow onset, confirming that activation does not necessarily require high shear stress once vWf can bind to GP Ib $\alpha$ (13). This response was, as expected, inhibited by blocking GP Ib $\alpha$ as well as $\alpha_{\mathrm{IIb}} \beta_{3}$ (Fig. 5). In sharp contrast, however, asialo-vWf not only failed to promote interplatelet cohesion if the shear rate was increased to $10,800 \mathrm{~s}^{-1}$, but also markedly inhibited the expected aggregation mediated by native vWf (Fig. 5). Such an effect was not due to any change in the ability to interact with stimulated $\alpha_{\mathrm{IIb}} \beta_{3}$, since asialo-vWf was equal to or better than the native molecule in this regard (not shown). Moreover, the effect was dose-dependent, and decreasing asialo vWf concentrations resulted in progressively less aggregation at a lower shear rate as well as less inhibition of aggregation at a higher shear rate (not shown). It is apparent, therefore, that the role of vWf in platelet aggregation under conditions of high shear rate is not likely to be predominantly dependent on its ability to act as an agonist after binding to GP Ib $\alpha$ (8).

Influence of shear rate on ligand binding to activated platelets. To evaluate whether the effects of shear forces on aggregation were the consequence of changes in ligand binding, fibrinogen and $\mathrm{vWf}$ interaction with agonist-activated platelets was measured before and after exposure to shearing flow, with

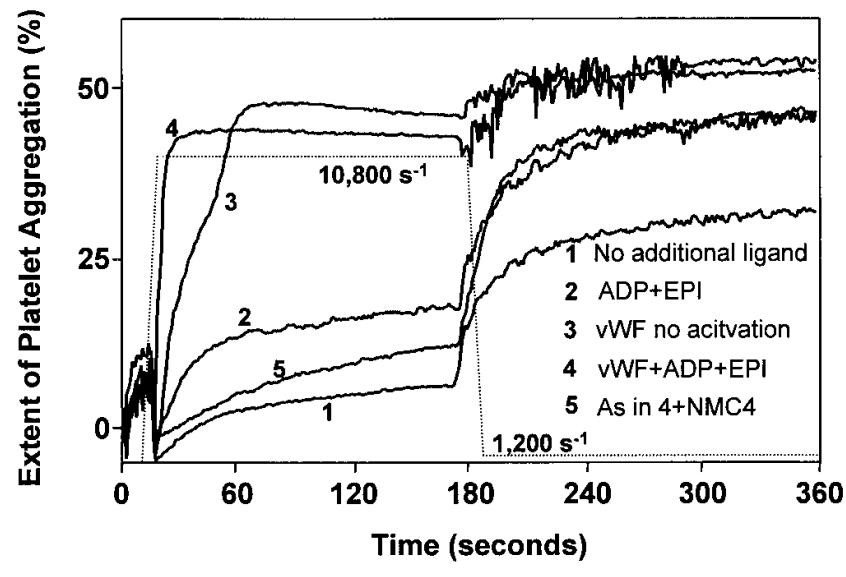

Figure 4. Effect of added soluble vWf on washed platelet aggregation at different shear rates. These experiments were performed with washed platelets prepared as described in the legend to Fig. 3, without added exogenous agonists (traces 1 and 3 ) or after activation with the combination of ADP and epinephrine, $20 \mu \mathrm{M}$ each (traces 2, 4, and 5). When indicated, vWf was added at the final concentration of $15 \mu \mathrm{g} / \mathrm{ml}$ (traces 3, 4 and 5), or replaced by an equal volume of Hepes-Tyrode buffer, $\mathrm{pH}$ 7.4. The anti-A1 domain antibody NMC-4 was used as Fab fragment at the final concentration of $500 \mu \mathrm{g} / \mathrm{ml}$. The specificity of its effect on vWf binding to platelets under the influence of shear stress has been documented previously (16). The transition from higher to lower shear rate was attained as described in the legend to Fig. 3. 

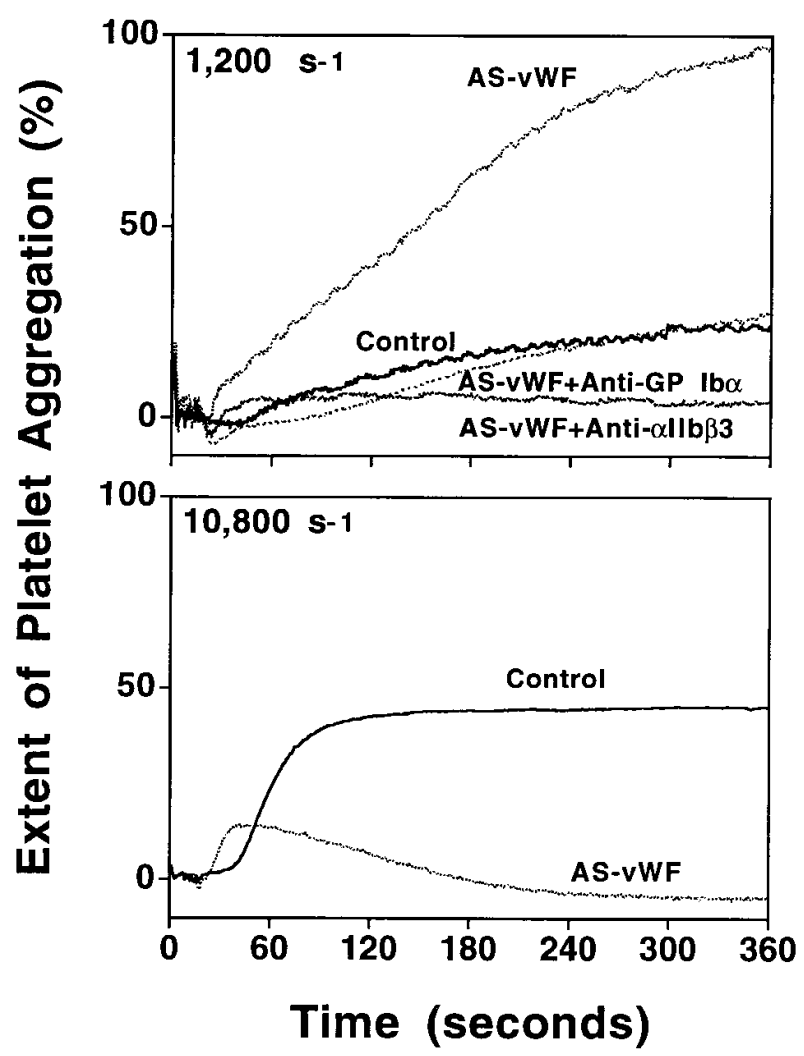

Figure 5. Shear stress-induced platelet aggregation in the presence of asialo-vWf. The experiments were performed at either $1,200 \mathrm{~s}^{-1}$ or $10,800 \mathrm{~s}^{-1}$ as indicated, using platelet-rich plasma to which asialo-vWf (final concentration $40 \mu \mathrm{g} / \mathrm{ml}$ ) was added immediately before exposure to shear forces. Control experiments were performed using modified Hepes-Tyrode buffer, $\mathrm{pH} 7.4$, instead of asialo-vWf. Monoclonal antibodies against GP Ib $\alpha$ and $\alpha_{\text {II }} \beta_{3}$ were used as described in the legend to Fig. 1. The results presented here are representative of two experiments performed with blood from different donors that gave comparable results.

or without specific receptor blockade. Control and anti-GP Ib $\alpha$-treated platelets exhibited similar binding of both ligands, but single platelets with maximum numbers of bound ligand molecules were markedly reduced in both instances after exposure to either low $\left(1,200 \mathrm{~s}^{-1}\right)$ or high $\left(10,800 \mathrm{~s}^{-1}\right)$ shear rate (Fig. 6). Although the latter finding may appear to indicate decreased average receptor occupancy in the platelet population exposed to shearing flow, it is actually more likely to be an indirect sign of the continued presence of adhesive proteins bound to $\alpha_{\mathrm{II}} \beta_{3}$ on both control and anti-GP Ib $\alpha$ treated platelets. This apparent paradox can be explained by noting the following: (a) both fibrinogen and vWf could promote aggregation of platelets exposed to low shear rate by binding directly to activated $\alpha_{\mathrm{IIb}} \beta_{3}$ (Fig 3); (b) transition to low shear rate allowed aggregation of control as well as GP Ib $\alpha$-blocked platelets, even after exposure to a high shear rate (Fig. 3; such a transition always occurred during the experiments shown in Fig. 6 when the device used to impose fluid motion was stopped to measure platelet-bound ligand); and (c) aggregates were excluded from the flow cytometric analysis used for these studies since gating parameters for fluorescent particles were set on the size of single platelets, a necessary requirement for meaningful quantitative evaluation of ligand binding. Consequently, because platelets with $\alpha_{\mathrm{IIb}} \beta_{3}$-bound fibrinogen or vWf would be expected to aggregate during the course of the experiments shown in Fig. 6, the results for control and anti-GP Ib $\alpha$-treated platelets may represent only the population that was not incorporated into aggregates, i.e., mostly with lower levels of receptor occupancy. In contrast, activated platelets treated with the anti- $\alpha_{\mathrm{IIb}} \beta_{3}$ antibody exhibited markedly reduced receptor occupancy by fibrinogen or vWf whether or not they were exposed to high or low shear rate (Fig. 6), in agreement with their impaired ability to aggregate at any shear rate tested (Fig. 3). Altogether, it is apparent that shear stress has little influence on ligand binding to activated $\alpha_{\text {IIb }} \beta_{3}$. All the results presented so far, therefore, are compatible with the conclusion that platelets can be linked to one another by adhesive proteins interacting solely with $\alpha_{\mathrm{II}} \beta_{3}$ if fluid dynamic forces are relatively low, whereas optimal aggregation at higher shear rate levels requires the function of vWf and GP Ib $\alpha$, even when $\alpha_{\mathrm{IIb}} \beta_{3}$ is fully activated and occupied by competent ligands.

Role of $v W f$ and GP Ib $\alpha$ in platelet thrombus development under flow conditions. To obtain comparable initial surface coverage for all conditions to be tested in these experiments and to eliminate the influence of platelet-substrate interactions, thrombus formation was initiated by perfusing a constant volume of normal blood over collagen type I fibrils at shear rate of $1,500 \mathrm{~s}^{-1}$ for $1 \mathrm{~min}$ (26). This perfusion was immediately followed by an aliquot of the same blood, either untreated or containing specific monoclonal antibodies, perfused at the same shear rate for additional $5 \mathrm{~min}$. The sum of the cross-sectional area of thrombi, measured in planes at 5- $\mu \mathrm{m}$ intervals in the $z$ axis during uninterrupted flow, was markedly increased at the end of the second perfusion period with control blood (Fig. 7). The changes occurring in planes located at more than $5 \mu \mathrm{m}$ from the surface reflected greater thrombus height because of platelet-platelet interactions, and were completely inhibited by blocking either GP Ib $\alpha$ or $\alpha_{\text {IIb }} \beta_{3}$ (Fig. 7). These results, therefore, demonstrated that both receptors are necessary (and neither one sufficient) to support platelet aggregation under conditions that may mimic those in vivo. Indeed, shear rate values between 500 and $5,000 \mathrm{~s}^{-1}$ have been measured during normal circulation in arterioles of $10-50-\mu \mathrm{m}$ diameter (29). In vessels of this caliber, as in perfusion chambers, blood is assumed to behave as a Newtonian fluid with steady laminar flow not influenced by the cardiac cycle.

\section{Discussion}

We show here that aggregation of platelets moving with a high velocity differential requires the concurrent function of GP Ib $\alpha$ and $\alpha_{\text {IIb }} \beta_{3}$ as well as vWf as adhesive ligand. Such a mechanism of interplatelet contact is evoked at higher shear rates in the cone-and-plate viscometer where aggregates form in suspension, than in the perfusion chamber where thrombi grow anchored to a surface. This difference may indicate that there is a lower level of stress on adhesive bonds forming between freely moving platelets that can accelerate their motion upon attaching to faster ones (Fig. 8) as compared with bonds tethering flowing platelets to others already immobilized. Consequently, the shear rate needed to elicit specific platelet functions in vivo may be overestimated by studying the process of aggregation in suspension $(11,12,16)$. 


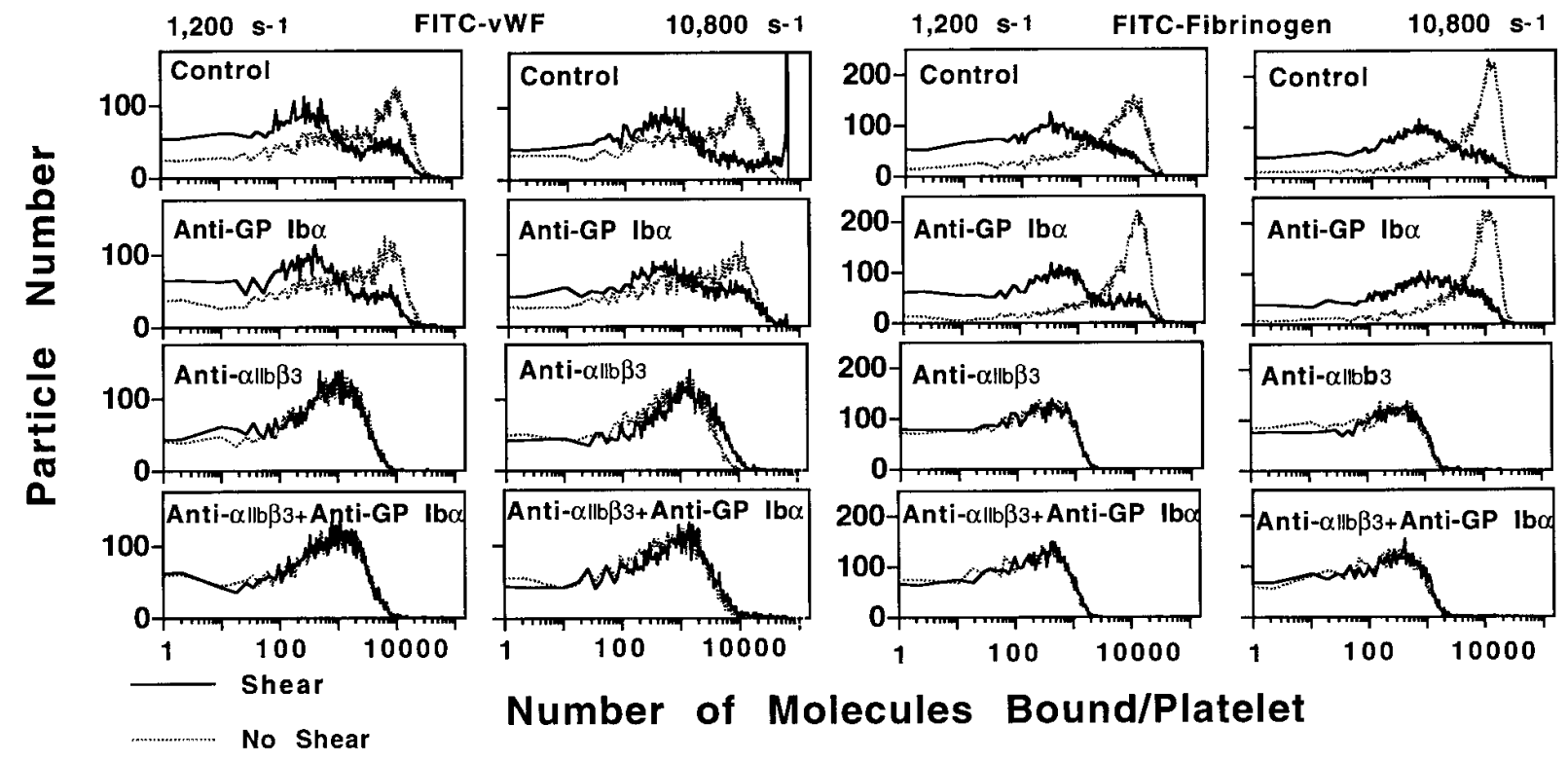

Figure 6. Platelet binding of FITC-labeled vWf and fibrinogen analyzed by flow cytometry. Experimental mixtures were prepared as described in the legend to Fig. 3, and were incubated at room temperature for $10 \mathrm{~min}$. They were then either exposed to different constant shear rates as indicated, or kept unstirred for the same period of time. At the end, $100 \mu \mathrm{l}$ of each sample was diluted with $400 \mu l$ of Hepes-Tyrode buffer to measure the number of ligand molecules bound per platelet (vWf subunit or intact fibrinogen) using a flow cytometer (16). Each experiment was repeated at least twice, with comparable results.

This notwithstanding, experimental models that establish a velocity differential between platelets in adjacent planes (Fig. 8) emulate conditions in the circulation (28), where flow limits the time available for receptor-ligand coupling. It has been shown previously that only GP Ib $\alpha$ binding to immobilized vWf can mediate platelet attachment to surfaces under rapid flow. This interaction is favored by a fast forward rate with high resistance to tensile stress, but is transient owing to a high dissociation rate (15). In contrast, interactions involving $\alpha_{\mathrm{IIb}} \beta_{3}$ are almost immediately irreversible, but of limited efficacy in high velocity shearing flow because of the relatively slow forward rate. These distinct properties synergistically support stable platelet adhesion because the initially transient tethers created by GP Ib $\alpha$ binding to vWf prolong the time available for activation and formation of bonds mediated by $\alpha_{\mathrm{IIb}} \beta_{3}$ (15). Our results now indicate that vWf and GP Ib $\alpha$ are similarly required to support $\alpha_{\text {IIb }} \beta_{3}$ function in linking one platelet to another at high shear rates.

It is commonly thought that agonist-dependent stimulation needed for soluble ligand binding to $\alpha_{\mathrm{IIb}} \beta_{3}$ must precede platelet involvement in aggregation. The data presented here, however, support the different model depicted schematically in Fig. 8, suggesting that stimulated platelets with membranebound adhesive proteins are an appropriate surface for attachment of additional platelets, whether the latter are activated or not. Indeed, the concepts proven valid for adhesion (15) seem to justify this model of aggregation, at least at high shear rates, since GP Ib $\alpha$ and vWf may promote initial interplatelet bonding regardless of activation. Accordingly, nonactivated $\alpha_{\text {IIb }} \beta_{3}$ may contribute to this function at lower shear rates by interacting selectively (30) with immobilized fibrinogen or fibrin (15). The newly recruited platelets are then activated by locally released or generated agonists, as well as by the effect of shear stress itself (28), so that their aggregation becomes irreversible and soluble ligands bind to their membranes, forming the substrate for continuation of the process (Fig. 8). This model of aggregation may better explain thrombus formation in vessels, since only platelets already attached at sites of injury (not those being recruited from circulating blood) are likely to have been stimulated by locally generated agonists.

The higher plasma concentration $\left(\sim 8 \times 10^{-6} \mathrm{M}\right)$ appears to favor fibrinogen for interacting with activated platelets (31). Nevertheless, vWf ( $\sim 2 \times 10^{-8} \mathrm{M}$ in plasma) may become immobilized onto the platelet membrane at adequate functional levels owing to increased concentrations in the local microenvironment after $\alpha$-granule release, but also as a consequence of shear stress-induced mechanisms that, independently of other stimuli, promote its binding in a process requiring both GP Ib $\alpha$ and $\alpha_{\mathrm{IIb}} \beta_{3}$ (16). Indeed, our findings (Fig. 2) indicate that, depending on the mechanism of activation, either fibrinogen or vWf can become the $\alpha_{\text {IIb }} \beta_{3}$-bound adhesive ligand, supporting aggregation at high shear rates. Binding of vWf may occur preferentially as a result of shear stress-induced activation because the latter process depends on an initial interaction of vWf itself with GP Ibo $(9,10)$. Thus, the changes that render $\alpha_{\text {II }} \beta_{3}$ capable of interacting with soluble ligands may involve only the platelets onto which vWf is already immobilized, albeit perhaps transiently (16), effectively increasing the local concentration of this ligand and favoring its binding to $\alpha_{\mathrm{IIb}} \beta_{3}$ over that of fibrinogen. In contrast, the presence of exogenous agonists in solution is likely to lead to $\alpha_{\mathrm{IIb}} \beta_{3}$ activation even on platelets that have not previously interacted with $\mathrm{vWf}$ through GP Ib $\alpha$, so that the higher concentration in plasma becomes the factor leading to preferential fibrinogen rather than vWf binding to $\alpha_{\mathrm{II}} \beta_{3}$. In any case, it is apparent that aggregation at high shear rates is always strictly dependent on $\mathrm{vWf}$ interaction with GP Ib $\alpha$, regardless of the nature of the $\alpha_{\text {IIb }} \beta_{3}$ activation process and even when fibrinogen is the adhesive 


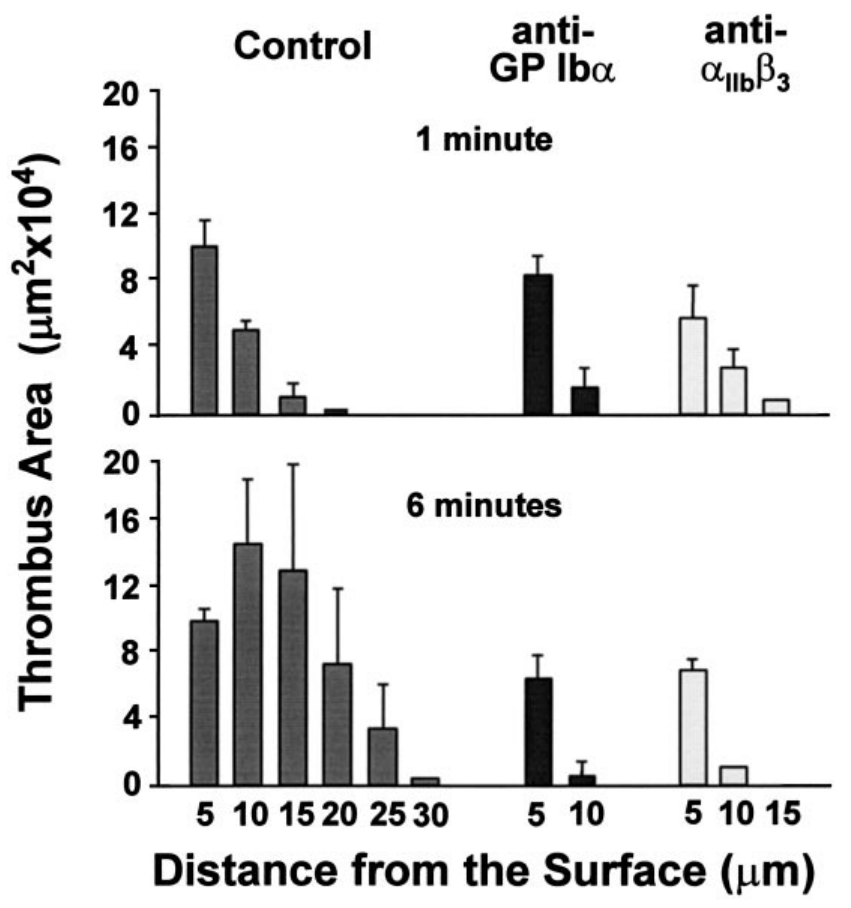

Figure 7. Effect of specific monoclonal antibodies on thrombus development in flowing blood. Blood containing $40 \mu \mathrm{M}$ PPACK as anticoagulant was aspirated through a parallel plate flow chamber (15) at a constant flow rate calculated to give an initial wall shear rate of $1,500 \mathrm{~s}^{-1}$. Cumulative cross sectional area occupied by mepacrinelabeled platelets in planes positioned at the indicated distance from the collagen type I-coated surface after 1 min of perfusion with normal blood (top); cumulative cross-sectional thrombus areas after uninterrupted continuation of the perfusion for additional $5 \mathrm{~min}$ with blood containing anti-GP Ib $\alpha$ or anti- $\alpha_{\mathrm{IIb}} \beta_{3}$ monoclonal antibody, as indicated, or $0.04 \mathrm{M}$ phosphate buffer, $\mathrm{pH} 7.4$, with $0.15 \mathrm{M} \mathrm{NaCl}$ in the control (bottom). Antibodies were used as described in the legend to Fig. 1, with concentrations in plasma calculated assuming a packed red cell volume of $45 \%$ of the total blood volume. Note that the plane $5 \mu \mathrm{m}$ from the surface shows similar platelet coverage under all experimental conditions, with minimal difference after 1 or 6 min of perfusion, indicating that adhesion to collagen was essentially complete at the end of the 1-min perfusion with untreated normal blood. The results shown are the mean $\pm \mathrm{SE}$ of the mean of five separate experiments performed with blood from different donors.

ligand bound to the latter receptor. Moreover, our findings (Fig. 4) indicate that this function is likely to be predominantly related to plasma rather than platelet vWf.

The mechanistic role proposed here for $\mathrm{vWf}$ and GP Ib $\alpha-$ to mediate interplatelet cohesion not dependent on $\alpha_{\mathrm{II}} \beta_{3}-$ differs substantially from that envisioned in the presence of ristocetin or botrocetin, and expands the concepts derived from the study of shear stress-induced aggregation. While promoting activation may be relevant in specific pathophysiological conditions (28), it appears that the vWf-GP Ib $\alpha$ interaction has a more general function in supporting interplatelet bridging under high flow conditions, providing adhesive properties synergistic with those of $\alpha_{\mathrm{II}} \beta_{3}$. The results obtained with asialovWf support this concept since enhanced binding to GP Ib $\alpha$, favoring receptor saturation on individual platelets (13), was detrimental for aggregation at higher shear rates in spite of the

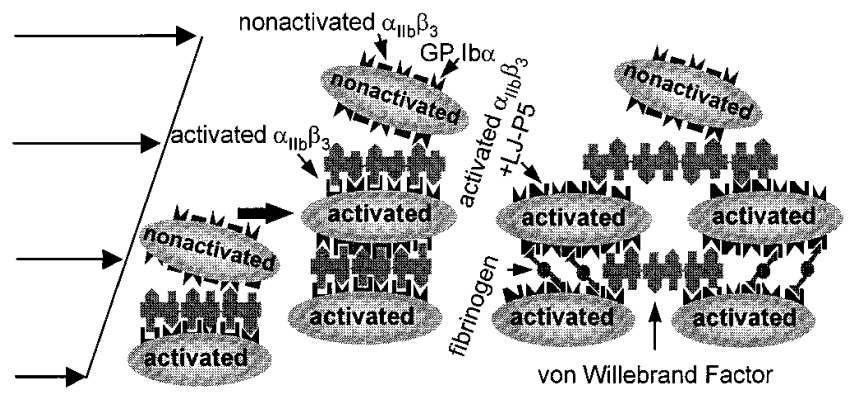

Figure 8. Schematic representation of the mechanisms of platelet aggregation in shearing flow. Platelets in adjacent planes of a fluid moving with laminar flow have different velocity. In the case of the coneand-plate viscometer depicted here, the velocity increases linearly with the distance from the stationary plate at the bottom (arrows). The linear slope of the velocity profile indicates that the shear rate, directly proportional to the speed of the rotating cone, is constant in all points of the moving fluid. In a parallel plate perfusion chamber, in contrast, the velocity profile is parabolic and the shear rate decreases progressively from the wall to the center of the lumen (not shown here). In a high velocity flow field, only GP Ib $\alpha$ interaction with vWf multimers immobilized onto the surface of other platelets can initiate interplatelet attachment irrespective of the activation state of $\alpha_{\text {II }} \beta_{3}$. This GP Ib $\alpha$-dependent interaction supports initially transient tethering, depicted by the ongoing detachment of the two top platelets from vWf multimers bound to already activated platelets. The process is amplified by the activation of $\alpha_{\mathrm{II}} \beta_{3}$ that may occur during the transient tethering, resulting eventually in stable attachment of recruited platelets and irreversible membrane binding of soluble adhesive ligands (16), thus providing the substrate for additional recruitment of nonactivated platelets. Note that nonactivated $\alpha_{\text {II }} \beta_{3}$ is assumed not to be able to bind soluble ligands. Activated $\alpha_{\mathrm{IIb}} \beta_{3}+$ LJ-P5 depicts a receptor-binding pocket that cannot interact with vWf, but can bind fibrinogen. The bridging effect of the latter adhesive protein can contribute to stabilizing platelet aggregation, but this function can only take place after the initial tethering supported by the vWf-GP Ib $\alpha$ interaction (see results in Fig. 2).

ability to cause effective activation. In this regard, asialo-vWf may act like an anti-GP Ib $\alpha$ antibody preventing tethering to vWf molecules immobilized onto the membrane of adjacent platelets. The synergism of ligand-receptor interactions with different kinetic properties demonstrated here for platelet aggregation in response to varying flow conditions may apply to other situations in which homotypic and heterotypic contacts occur between cells in circulating blood. Such may be the case in pathophysiological processes mediating immune response (32), inflammation (33), and allergic reactions involving eosinophils (34) and basophils (35). Similarly, lymphocytes may acquire adhesive properties mediated by platelet receptors after forming heterotypic aggregates (36), so that the synergism of distinct adhesion pathways may benefit cells originally not endowed with specific recognition specificities.

In conclusion, our findings support a novel interpretation of the mechanisms of platelet aggregation, highlighting the essential role of vWf and GP Ib $\alpha$ in response to varying hemodynamic conditions. This is likely to be important for normal hemostasis, but even more prominent in situations relevant to thrombosis since wall shear rates of 3,000-10,000 s${ }^{-1}$ have been measured at the top of plaques causing $50 \%$ occlusion of coronary arteries (37). It should be possible, therefore, to inhibit 
thrombus formation selectively in areas of the circulation where flow generates higher shear rates by blocking vWf binding to GP Ib $\alpha$, and to accomplish more effective global inhibition, when required, by dual receptor blockade. These considerations may support the evaluation of new anti-thrombotic strategies.

\section{Acknowledgments}

This work was supported by National Institutes of Health grants HL 31950, HL 42846, and HL 48728, and by the Stein Endowment Fund (to Z.M. Ruggeri). Support from National Institutes of Health grant RR00833 to the General Clinical Research Center of The Scripps Research Institute is also acknowledged. S. Goto received a travel grant from Tokai University School of Medicine Research Aid 1996.

\section{References}

1. Weiss, H.J. 1975. Platelet physiology and abnormalities of platelet function (first of two parts). N. Engl. J. Med. 293:531-541.

2. Weiss, H.J. 1975. Platelet physiology and abnormalities of platelet function (second of two parts). N. Engl. J. Med. 293:580-588.

3. Fuster, V., L. Badimon, J.J. Badimon, and J.H. Chesebro. 1992. The pathogenesis of coronary artery disease and the acute coronary syndromes (1). N. Engl. J. Med. 326:242-250

4. Fuster, V., L. Badimon, J.J. Badimon, and J.H. Chesebro. 1992. The pathogenesis of coronary artery disease and the acute coronary syndromes (2). N. Engl. J. Med. 326:310-318

5. Lefkovits, J., E.F. Plow, and E.J. Topol. 1995. Platelet glycoprotein IIb/ IIIa receptors in cardiovascular medicine. N. Engl. J. Med. 332:1553-1559.

6. Howard, M.A. and B.G. Firkin. 1971. Ristocetin - a new tool in the investigation of platelet aggregation. Thromb. Haemostasis. 26:362-369.

7. Read, M.S., S.V. Smith, M.A. Lamb, and K.M. Brinkhous. 1989. Role of botrocetin in platelet agglutination: formation of an activated complex of botrocetin and von Willebrand factor. Blood. 74:1031-1035.

8. Kroll, M.H., T.S. Harris, J.L. Moake, R.I. Handin, and A.I. Schafer. 1991. von Willebrand Factor binding to platelet GPIb initiates signals for platelet activation. J. Clin. Invest. 88:1568-1573.

9. Chow, T.W., J.D. Hellums, J.L. Moake, and M.H. Kroll. 1992. Shear stress-induced von Willebrand factor binding to platelet glycoprotein Ib initiates calcium influx associated with aggregation. Blood. 80:113-120.

10. Ikeda, Y., M. Handa, T. Kamata, K. Kawano, Y. Kawai, K. Watanabe, K. Sakai, F. Mayumi, I. Itagaki, A. Yoshioka, and Z.M. Ruggeri. 1993. Transmembrane calcium influx associated with von Willebrand factor binding to GP $\mathrm{Ib}$ in the initiation of shear-induced platelet aggregation. Thromb. Haemostasis. 69:496-502.

11. Peterson, D.M., N.A. Stathopoulos, T.D. Giorgio, J.D. Hellums, and J.L. Moake. 1987. Shear-induced platelet aggregation requires von Willebrand factor and platelet membrane glycoproteins Ib and IIb-IIIa. Blood. 69:625-628.

12. Ikeda, Y., M. Handa, K. Kawano, T. Kamata, M. Murata, Y. Araki, H. Anbo, Y. Kawai, K. Watanabe, et al. 1991. The role of von Willebrand Factor and fibrinogen in platelet aggregation under varying shear stress. J. Clin. Invest. 87:1234-1240.

13. De Marco, L., A. Girolami, S. Russell, and Z.M. Ruggeri. 1985. Interaction of asialo von Willebrand factor with glycoprotein $\mathrm{Ib}$ induces fibrinogen binding to the glycoprotein IIb/IIIa complex and mediates platelet aggregation. J. Clin. Invest. 75:1198-1203.

14. De Marco, L., A. Girolami, T.S. Zimmerman, and Z.M. Ruggeri. 1985. Interaction of purified IIB von Willebrand factor with the platelet membrane glycoprotein Ib induces fibrinogen binding to the glycoprotein IIb/IIIa complex and initiates aggregation. Proc. Natl. Acad. Sci. USA. 82:7424-7428.

15. Savage, B., E. Saldivar, and Z.M. Ruggeri. 1996. Initiation of platelet adhesion by arrest onto fibrinogen or translocation on von Willebrand factor. Cell. 84:289-297.

16. Goto, S., D.R. Salomon, Y. Ikeda, and Z.M. Ruggeri. 1995. Characterization of the unique mechanism mediating the shear-dependent binding of sol- uble von Willebrand factor to platelets. J. Biol. Chem. 270:23352-23361.

17. Ruggeri, Z.M., L. De Marco, L. Gatti, R. Bader, and R.R. Montgomery. 1983. Platelets have more than one binding site for von Willebrand factor. $J$ Clin. Invest. 72:1-12.

18. Niiya, K., E. Hodson, R. Bader, V. Byers-Ward, J.A. Koziol, E.F. Plow, and Z.M. Ruggeri. 1987. Increased surface expression of the membrane glycoprotein IIb/IIIa complex induced by platelet activation. Relationship to the binding of fibrinogen and platelet aggregation. Blood. 70:475-483.

19. Savage, B., S.J. Shattil, and Z.M. Ruggeri. 1992. Modulation of platelet function through adhesion receptors: A dual role for glycoprotein IIb-IIIa (integrin $\alpha_{I I b} \beta_{3}$ ) mediated by fibrinogen and glycoprotein Ib-von Willebrand factor. J. Biol. Chem. 267:11300-11306.

20. Trapani-Lombardo, V., E. Hodson, J.R. Roberts, T.J. Kunicki, T.S. Zimmerman, and Z.M. Ruggeri. 1985. Independent modulation of von Willebrand factor and fibrinogen binding to the platelet membrane glycoprotein IIb/ IIIa complex as demonstrated by monoclonal antibody. J. Clin. Invest. 76:19501958

21. Weiss, H.J., J. Hawiger, Z.M. Ruggeri, V.T. Turitto, P. Thiagarajan, and T. Hoffmann. 1989. Fibrinogen-independent platelet adhesion and thrombus formation on subendothelium mediated by glycoprotein IIb-IIIa complex at high shear rate. J. Clin. Invest. 83:288-297.

22. Fujimura, Y., Y. Usami, K. Titani, K. Niinomi, K. Nishio, T. Takase, A. Yoshioka, and H. Fukui. 1991. Studies on anti-von Willebrand factor (vWf) monoclonal antibody NMC-4, which inhibits both ristocetin- and botrocetininduced vWf binding to platelet glycoprotein Ib. Blood. 77:113-120,

23. Shelton-Inloes, B.B., K. Titani, and J.E. Sadler. 1986. cDNA sequences for human von Willebrand factor reveal five types of repeated domains and five possible protein sequence polymorphisms. Biochemistry. 25:3164-3171.

24. Fujimura, Y., K. Titani, L.Z. Holland, S.R. Russell, J.R. Roberts, J.H. Elder, Z.M. Ruggeri, and T.S. Zimmerman. 1986. von Willebrand factor. A reduced and alkylated 52/48 $\mathrm{kDa}$ fragment beginning at amino acid residue 449 contains the domain interacting with platelet glycoprotein Ib. J. Biol. Chem. 261:381-385.

25. Miyata, S., S. Goto, A.B. Federici, J. Ware, and Z.M. Ruggeri. 1996 Conformational changes in the A1 domain of von Willebrand factor modulating the interaction with platelet glycoprotein Ib $\alpha$. J. Biol. Chem. 271:9046-9053.

26. Alevriadou, B.R., J.L. Moake, N.A. Turner, Z.M. Ruggeri, B.J. Folie, M.D. Phillips, A.B. Schreiber, M.E. Hrinda, and L.V. McIntire. 1993. Real-time analysis of shear-dependent thrombus formation and its blockade by inhibitors of von Willebrand factor binding to platelets. Blood. 81:1263-1276.

27. Moake, J.L., N.A. Turner, N.A. Stathopoulos, L. Nolasco, and J.D. Hellums. 1988. Shear-induced platelet aggregation can be mediated by vWf released from platelets, as well as by exogenous large or unusually large $\mathrm{vWf}$ multimers, requires adenosine diphosphate, and is resistant to aspirin. Blood. 71: 1366-1374.

28. Kroll, M.H., J.D. Hellums, L.V. McIntire, A.I. Schafer, and J.L. Moake. 1996. Platelets and shear stress. Blood. 88:1525-1541.

29. Tangelder, G.J., D.W. Slaaf, T. Arts, and R.S. Reneman. 1988. Wall shear rate in arterioles in vivo: least estimates from platelet velocity profiles. Am. J. Physiol. 254:H1059-H1064.

30. Savage, B., and Z.M. Ruggeri. 1991. Selective recognition of adhesive sites in surface-bound fibrinogen by GP IIb-IIIa on nonactivated platelets. $J$. Biol. Chem. 266:11227-11233.

31. Pietu, G., G. Cherel, G. Marguerie, and D. Meyer. 1984. Inhibition of von Willebrand factor-platelet interaction by fibrinogen. Nature. 308:648-649.

32. Springer, T.A. 1990. Adhesion receptors of the immune system. [Review]. Nature. 346:425-434.

33. Entman, M.L., L. Michael, R.D. Rossen, W.J. Dreyer, D.C. Anderson, A.A. Taylor, and C.W. Smith. 1991. Inflammation in the course of early myocardial ischemia. [Review]. FASEB J. 5:2529-2537.

34. Teixeira, M.M., T.J. Williams, B.T. Au, P.G. Hellewell, and A.G. Rossi 1995. Characterization of eosinophil homotypic aggregation. J. Leukocyte Biol. 57:226-234.

35. Knol, E.F., T.W. Kuijpers, F.P. Mul, and D. Roos. 1993. Stimulation of human basophils results in homotypic aggregation. A response independent of degranulation. J. Immunol. 151:4926-4933.

36. Diacovo, T.G., K.D. Puri, R.A. Warnock, T.A. Springer, and U.H. von Andrian. 1996. Platelet-mediated lymphocyte delivery to high endothelial venules. Science. 273:252-255.

37. Back, C.H., J.R. Radbill, and D.W. Crawford. 1977. Analysis of pulsatile viscous blood flow through diseased coronary arteries of man. J. Biomech. 10 339-353. 